\section{Cahiers de Narratologie}

Analyse et théorie narratives

31 | 2016

Sérialité narrative. Enjeux esthétiques et économiques

\title{
Introduction. Sérialité narrative : enjeux esthétiques et économiques
}

\section{Alessandro Leiduan}

\section{(2) OpenEdition}

\section{Journals}

Electronic version

URL: http://journals.openedition.org/narratologie/7561

DOI: 10.4000/narratologie.7561

ISSN: 1765-307X

Publisher

LIRCES

\section{Electronic reference}

Alessandro Leiduan, «Introduction. Sérialité narrative : enjeux esthétiques et économiques », Cahiers de Narratologie [Online], 31 | 2016, Online since 22 December 2016, connection on 23 September 2020. URL : http://journals.openedition.org/narratologie/7561; DOI : https://doi.org/10.4000/narratologie. 7561

This text was automatically generated on 23 September 2020 .

Article L.111-1 du Code de la propriété intellectuelle. 


\title{
Introduction. Sérialité narrative : enjeux esthétiques et économiques
}

\author{
Alessandro Leiduan
}

1 La notion de littérature a longtemps été l'otage d'une idéologie qui tendait à circonscrire ses effets sociaux à la sphère esthétique. Dans la tradition culturelle occidentale, celle-ci s'est affirmée comme le lieu par excellence d'un plaisir désintéressé ${ }^{1}$ : la méconnaissance des intérêts qui ont de tous temps présidé à l'interaction entre l'imaginaire social et les œuvres littéraires est ainsi devenue le trait distinctif du crédo esthétique moderne (Gadamer: $\left.1960^{2}\right)$. Au fur et à mesure que le capitalisme, sous l'égide des politiques libérales, s'imposait comme le seul et unique mécanisme régulateur de la vie sociale, le déni des intérêts extra-esthétiques s'est traduit par un reniement de la dimension économique de l'art (Bourdieu: 19983). Enjoliver l'apparence d'un récit pour en augmenter l'attrait commercial a été considéré comme une trahison des finalités sociales de ce type de pratique symbolique.

2 Quels ont été les effets de l'ingérence de l'économie sur les pratiques d'écriture narrative? Comment ont évolué les critères de réception sociale des récits sous l'effet de cette influence?

3 C'est autour de ces enjeux thématiques que s'est déroulé du 26 au 29 juillet 2015, à l'université de Mannheim, un colloque international de narratologie, réunissant des chercheurs issus de plusieurs universités européennes. Les Cahiers de narratologie consacrent ce numéro à la présentation des actes de ce colloque. L'étude des rapports entre l'esthétique et l'économie peut faire l'objet d'une multitude d'approches: les articles ici réunis éclairent le sujet à partir d'angles de lecture différents, mais gravitent tous autour d'un même thème fédérateur : la notion de sérialité. Dans un contexte social où tout - désormais - est voué à l'échange marchand, la forme sous laquelle les choses s'offrent à l'attention humaine est, en effet, celle d'un «objet sériel». Il s'agit là du corollaire inévitable du mode de fonctionnement du modèle économique occidental : le propre du capitalisme est de permettre à ceux qui investissent de l'argent dans un circuit économique de réaliser du profit afin de pouvoir réinvestir les bénéfices dans un autre cycle productif. À cet effet, le prix de vente d'une chose ne peut pas être inférieur à 
l'argent investi pour sa fabrication, mais il ne doit pas non plus être trop élevé par rapport aux capacités d'achat des consommateurs. Pour préserver la valeur marchande d'un produit, c'est-à-dire sa capacité à rencontrer des acheteurs solvables, l'industrie capitaliste a donc été contrainte d'adopter très rapidement un mode de production spécifique. La fabrication à la chaîne de grandes quantités de produits standardisés s'est vite imposée comme le mode de production le plus adapté à cette fin. Augmenter la quantité des biens produits permet, en effet, de réduire le coût unitaire de fabrication d'un produit. La contrepartie de ce mode de production est pourtant une certaine standardisation: les produits issus de la même chaîne de montage sont parfaitement identiques, tous ayant été fabriqués à partir du même prototype. Les objets industriels de qualité homogène (meubles, bibelots, voitures, appareils ménagers, etc.) qui remplissent notre vie quotidienne en sont l'illustration symptomatique. Les récits, à leur tour, sous l'effet des contraintes économiques qui régissent la production de tous les objets existants, n'échappent pas à ce phénomène d'homogénéisation.

Les lignes suivantes seront consacrées, en guise d'introduction à ce numéro, à l'exploration de cette notion de "sérialité élargie » à la lumière de laquelle il convient, selon nous, de repenser l'interaction entre la société et ses pratiques narratives. Il s'agira notamment d'expliquer (i) en quoi les récits sériels se différencient des autres objets sériels, (ii) en quoi un horizon d'attente peut être qualifié de " sériel », (iii) en quoi, enfin, les critères narratologiques qui président actuellement à l'analyse critique et à l'appréciation esthétique d'un récit peuvent être, à leur tour, qualifiés de « sériels » et considérés, au même titre, comme le signe d'une standardisation générale de l'imaginaire social.

\section{La « sérialité " comme mode de production}

Les séries narratives ne sont pas des collections de récits identiques les uns aux autres, racontant $n$ fois la même histoire sans que la moindre variation ne distingue la version $d u$ récit $n 1$ de celle des récits $n 2, n 3$, etc. Personne n'accepterait, par exemple, de voir deux fois le même épisode d'une série télé. Or, si les séries narratives étaient vraiment des équivalents des produits standardisés de l'industrie sérielle, les téléspectateurs devraient pouvoir se réjouir d'assister inlassablement au déroulement du même épisode. N'achètet-on pas des voitures, des vêtements, des appareils ménagers justement parce qu'ils sont conformes à un modèle standard? S'ils peuvent faire l'objet d'une appropriation personnelle, ces objets n'en sont pas moins tous identiques. Mais leur homogénéité sérielle ne nuit pas (loin s'en faut) à leur appétibilité commerciale. Les séries narratives sauraient-elles s'accommoder d'un degré de standardisation si élevé ? Bien sûr que non. Elles doivent impérativement s'appuyer sur une stratégie de diversification partielle de leurs contenus fixes. Toutes les séries narratives comportent ainsi un noyau diégétique fixe (la présence d'un/de plusieurs héros récurrent(s), d'un cadre spatial plus ou moins stable, d'un thème fédérateur commun); mais la présence de ces invariants est contrebalancée par des variations destinées à introduire dans chaque récit de l'imprévu, de manière à donner l'impression que chaque nouvel épisode diffère du précédent, alors que le schéma narratif demeure identique ${ }^{4}$. Chaque récit sériel repose donc sur une combinatoire d'éléments variables et invariables: les uns, pourrait-on dire, servent à "dissimuler» les autres. Le propre de ce type de récit est de donner au public l'impression de découvrir à chaque occasion une histoire nouvelle (qui, en réalité, est 
toujours plus ou moins identique), alors même que le plaisir qu'éprouve le public naît précisément de l'itération d'un même procédé narratif (Benassi 2000 : 81). Les paramètres de différenciation peuvent être d'ordre sémantique, spatial, temporel, narratif ou discursif. Cependant, bien que cette "différence» soit simplement de l'ordre de l'« illusion », cette « différence illusoire » représente bien la clé du succès des séries qui ne sauraient satisfaire les attentes de leur public si elles dérogeaient à l'obligation d'assortir de l'inconnu au connu, de l'instabilité à la stabilité.

6 La sérialité narrative a donc très peu de choses à voir avec la sérialité industrielle. Cette dernière vise la reproduction d'un prototype et subordonne la mise sur le marché d'un produit à son caractère homogène et standard. La sérialité narrative, quant à elle, est fondée sur une dialectique entre identité et altérité, continuité et rupture, invariants et variations.

\section{La « sérialité » comme mode de réception}

7 Un récit n'existe pas au même titre qu'un autre objet matériel - comme une table, un marteau, une voiture. Contrairement à tous ces objets qui existent indépendamment de la représentation mentale que nous en avons, l'existence du récit est, quant à elle, entièrement tributaire d'une opération mentale, celle qui consiste à produire cet « objet " et à l'interpréter. On pourrait même dire qu'une œuvre narrative n'existe pas véritablement en dehors de ces deux opérations qui représentent chacune des lieux d'actualisation de son être. Il ne faut donc pas confondre un récit avec le substrat matériel qui permet à son sens d'interagir avec l'imaginaire de la société. Mon exemplaire de Madame Bovary dans l'édition de poche Garnier-Flammarion n'est pas le roman de Flaubert : il est une simple "extériorisation » de son essence véritable, celle qui s'actualise lorsque j'accomplis les opérations mentales nécessaires à l'interprétation de son sens. S'il est vrai que, pour accomplir ce genre d'opérations, j'ai besoin de la médiation sémiotique d'un livre, il n'en demeure pas moins vrai que l'histoire renfermée dans ce roman ne se confond pas avec son moyen de diffusion (Pouivet 2003 : 24). La nature d'un récit est donc celle d'un objet immatériel. De ce fait, la sérialité narrative serait à rechercher du côté de l'interaction entre le sens d'un récit et l'imaginaire social. Envisagée ainsi, elle pourrait être définie comme le corrélat de la décision prise par l'interprète de déchiffrer une histoire à la lumière d'autres histoires, en raison des ressemblances d'ordre sémantique, spatial, temporel, narratif ou discursif qui permettent d'envisager ces histoires comme un système de variations autour d'un noyau diégétique fixe. Faute de quoi, les «épisodes » d'une série narrative perdraient leur caractère "sériel », l'interprète n'étant pas capable de saisir les rapports qui leur permettent de «faire système » autour d'un socle diégétique commun. Nous dirons donc, avec Marc Marti, que « la sérialité est tout autant un mode de production des objets culturels qu'un mode de réception ${ }^{5}$ » et que les formes les plus répandues de sérialité contemporaine doivent davantage leur existence à la manière dont les récits sont interprétés qu'à la manière dont ils sont produits. L'un des aspects les plus marquants de la culture narrative actuelle est celui qui consiste à « mettre en série » des objets qui, à l'origine, n'avaient pas été conçus dans cette perspective. Les éditeurs ont de plus en plus tendance à structurer leurs catalogues selon des séries thématiques (le genre policier est devenu, par exemple, la "série noire » chez Gallimard). Les téléfilms sont le plus souvent regroupés de manière à constituer un ensemble d'œuvres homogènes formant un système autour de personnages et/ou de thèmes communs (Benassi 2000). 
Plus en général, ce sont tous les récits fictionnels contemporains qui ont tendance à se structurer autour d'un noyau diégétique commun, servant de lien sériel entre les différents " épisodes " regroupés sous une étiquette générique (telle que "collection ", "catalogue», "genre»). Malgré la diversité des noms que l'on donne à ces regroupements de récits, il s'agit de « séries » à peine déguisées : l'élément fédérateur est soit un personnage (le détective Pepe Carvalho dans les romans de Manuel Vàsquez Montalbàn), soit un schéma narratif (l'intrigue policière des romans pour jeunes lecteurs de la série "Chair de poule»), soit un thème (les affaires criminelles des séries télé documentaires telles que "Faites entrer l'accusé », «Enquêtes criminelles ", « Suspect n. $\left.1^{6} »\right)$.

8 Aux côtés des "séries" proprement dites, articulant une même histoire à travers plusieurs "micro-récits ", tantôt sur un mode itératif (les séries bouclées), tantôt sur un mode fragmenté (les séries feuilleton), il faudra donc faire place à des « séries » d'un genre différent, dont l'existence dépend de l'horizon d'attente « sériel » à la lumière duquel la société contemporaine envisage désormais les pratiques narratives.

\section{La « sérialité » comme paradigme critique et esthétique}

9 C'est de plus en plus en termes «sériels "-nous venons de le voir - que les œuvres narratives sont étudiées et appréciées. Bien qu'en matière narrative aucune des approches critiques et esthétiques aujourd'hui dominantes ne se réclame de la notion de " sérialité », l'idée qui sous-tend l'ensemble des jugements critiques et esthétiques portés sur les œuvres narratives témoigne d'une adhésion implicite à cette notion.

10 Le propre d'un récit sériel, on l'a vu, est qu'il ne peut être reconnu en tant que tel qu'à la lumière d'une comparaison avec plusieurs autres récits. L'interprète se doit de le comparer à d'autres récits afin de saisir les similitudes et les différences à la lumière desquelles le récit en question peut être reconnu comme un avatar sériel de la narrativité, c'est-à-dire comme une combinaison de répétitivité et d'innovation, d'éléments invariables et d'éléments variables. Le récit acquiert alors le statut sériel par excellence : celui d'une variante. Dans une série, chaque récit est la variante d'un autre, la série étant, dans son ensemble, un réseau de variations. La reconnaissance de la sérialité présuppose donc un réflexe de lecture spécifique qui aujourd'hui se généralise pourtant à l'ensemble des œuvres narratives : loin d'être limité à la réception des récits sériels proprement dits, son domaine d'application s'étend désormais à l'ensemble de l'univers narratif. C'est, dès lors, l'horizon d'attente de toute la société à l'égard des œuvres narratives qui est devenu " sériel », faisant ainsi de la sérialité une catégorie descriptive coextensive de l'ensemble de la production narrative. Cet horizon d'attente s'est mis en place dans le courant des dernières décennies, à l'initiative des spécialistes universitaires de la littérature qui, soucieux de s'affranchir d'une approche herméneutique des faits littéraires, ont entrepris de redéfinir leur objet d'étude (la littérature) à la lumière des corrélations existantes entre une œuvre et une autre, à la lumière aussi de l'ensemble des conventions, des normes et des règles qui, à titres divers, façonnent l'identité générique, textuelle et ontologique des créations littéraires (Genette 1982). A l'aune de ce nouveau « contrat de lecture ", les récits ont été décrits comme un réseau de correspondances de type formel ou thématique, chaque œuvre narrative faisant écho aux topoi rhétoriques des autres 
œuvres, l'identité de chacune ne pouvant être saisie que "relationnellement », c'est-à-dire au moyen d'une comparaison avec d'autres œuvres (Bourdieu 1979: 36). «On écrit des livres uniquement sur d'autres livres ou autour d'autres livres» a écrit U. Eco (Eco 1983 : 522) : cette devise postmoderne est en consonance parfaite avec une vision « sérielle » des pratiques narratives (vision qui n'est autre - pourrait-on même dire - que la propagation d'un réflexe de lecture savant au-delà des cercles érudits).

\section{Enjeux esthétiques et économiques des séries narratives}

11 Il semblerait donc qu'il y a bien lieu de parler de "sérialité " à propos des récits contemporains. Mais ces nouvelles formes narratives "sérielles» témoignent-elles forcément d'une inféodation de l'ensemble de nos pratiques symboliques aux lois de l'économie?

Les réponses à ce sujet ne peuvent qu'être très variées.

13 Selon Raphaël Baroni, dans l'article qui inaugure ce recueil, les stratégies narratives qui, à l'instar du cliffhanger, permettent aux récits sériels d'intriguer leurs lecteurs ne doivent pas être assimilées à des mécanismes commerciaux, se limitant à susciter, à la fin de chaque épisode, l'intérêt du lecteur pour l'épisode suivant. Parce qu'elles " plongent le lecteur au cœur du déroulement d'un événement encore inachevé », les stratégies narratives qui président au succès des récits sériels remplissent également d'autres fonctions anthropologiques. Elles permettent notamment aux lecteurs d'acquérir une meilleure compréhension de l'agir humain, dans la mesure où les situations à l'issue incertaine qu'ils découvrent dans un récit sont ni plus ni moins celles qu'ils affrontent dans leur existence quotidienne. La mise en scène d'un monde marqué par une indétermination provisoire ou définitive place, en effet, le lecteur dans une posture propice à la planification d'une action responsable, dès lors que le futur apparaît sous la forme de potentialités encore ouvertes. La narration - y compris sous sa forme " sérielle »- peut ainsi contribuer à exercer et affiner, sur le mode de l'« exemplification modélisante » (Schaeffer 1999), les réflexes comportementaux qui président au développement d'une conception responsable de l'agir éthique et politique.

14 Anaïs Goudmand étudie un procédé d'écriture généralement connoté comme commercial : la publication de romans en tranches quotidiennes dans les colonnes d'un journal afin d'attirer un lectorat plus large et de garantir aux annonceurs un retour sur investissement satisfaisant. En tant que prototype historique des formes de sérialité contemporaines, le roman feuilleton repose sur des stratégies récurrentes qui permettent de stimuler et de maintenir l'intérêt du lecteur (par exemple : le cliffhanger). Pourtant, sa raison d'être n'est pas exclusivement commerciale: les lecteurs des romans feuilletons tendent de fait à interpréter les romans feuilletons sur un mode qui n'est pas si éloigné de celui qui caractérise la compréhension de leur expérience vécue, telle qu'ils l'éprouvent au quotidien.

15 Les récits sériels ne relèvent pas seulement du domaine fictionnel, mais aussi du domaine factuel. Selon Marie Vanoost, le journalisme narratif est un modèle "relativement flexible de sérialité de type générique » qui s'organise autour d'une notion "intrigante » non pas (seulement) "configurante» de la diégésis. Les procédés d'écriture qui caractérisent ce type de journalisme participent, bien entendu, de la volonté d'attirer des 
lecteurs dans l'espoir de les fidéliser. Mais leur raison d'être n'est pas seulement économique, car ils témoignent aussi de l'ambition de repenser la pratique journalistique au-delà des codes déontologiques (l'identité professionnelle), éthiques et stylistiques qui ont gouverné jusqu'ici le journalisme « classique ». S'ils offrent une expérience de lecture proche de celle d'un récit fictionnel, les récits journalistiques rassurent les lecteurs sur le caractère réel et vérifié des événements racontés et favorisent une compréhension de la réalité événementielle qui, dans les intentions de leurs auteurs, devrait être plus profonde et pénétrante que celle que l'on peut escompter des récits journalistiques traditionnels.

Françoise Revaz prend en examen, dans son article, un genre prototypique de la littérature d'imagination à vocation commerciale, à savoir la série de BD. Selon un préjugé largement répandu, le propre de la littérature bédéique serait de proposer des aventures au fort impact émotionnel, dans le but de fidéliser les lecteurs à ce genre de loisirs imaginaires, dans le but aussi d'assurer de confortables revenus aux producteurs de ce type d'histoires. Or, l'enjeu de l'article est de montrer que, sous les apparences d'une histoire presque invariable, reproduisant d'épisode en épisode le même schéma narratif, la série Little Sammy Sneeze, de Winsor McCay, développe également une critique sociale du mode de vie de la société nord-américaine des années 1900, de ses activités routinières et de ses échanges verbaux vides de sens. Ce constat met en échec la distinction dichotomique entre, d'une part, une littérature dite « d'imagination » dont le seul but serait de proposer au lecteur des aventures divertissantes et, d'autre part, une littérature « factuelle » qui aurait le monopole du questionnement social et politique.

La transposition en $\mathrm{BD}$ de la Recherche proustienne semble être un pari impossible à relever. Lorsque Stéphane Heuet s'est attelé à cette tâche les critiques ont éreinté son initiative, n'y reconnaissant qu'une simple opération de merchandising. Julie Müller, dans son article "Proust en $\mathrm{BD}$ : économie de lecture et ré-création proustienne", prend le contrepied de ces critiques et montre que les principes d'économie qui régissent l'écriture des $\mathrm{BD}$ ne sont pas incompatibles avec une réception esthétique des livres de $\mathrm{S}$. Heuet.

18 Marc Marti reconstruit, dans son article, l'évolution du concept de " sérialité » : celle-ci n'est plus aujourd'hui le simple corrélat du mode de production des industries culturelles, mais recouvre des pratiques diverses d'interconnexion de récits qui, à l'origine, n'avaient pas été conçus pour former une "série ». Parmi ces nouvelles formes de sériation, le storytelling transmédia reproduit un même contenu diégétique sur une multiplicité de supports sémiotiques et médiatiques. L'objectif commercial qui préside à ces différentes formes de sérialité ne saurait éclipser leurs enjeux esthétiques, éthiques et politiques. Pour donner la mesure de ces enjeux, Marti étudie un jeu vidéo (du genre FPS) développé par un groupe de fans espagnols, España en llamas, qui se présente comme une réadaptation d'un grand classique de jeu de tir à la première personne, Call of Duty. Si le cadre historique demeure le même (la deuxième guerre mondiale), la version espagnole choisit de situer le déroulement des combats autour des campagnes militaires qui ont caractérisé la guerre d'Espagne. Le souci d'éviter toute polémique idéologique autour de cette guerre est à l'origine d'une modélisation relativement « consensuelle » de la réalité historique qui, sans être complètement " décalée », ne reproduit qu'en partie seulement la condition sociale, l'identité nationale ou le crédo politique des forces engagées dans les combats. Les enjeux politiques - on le voit - priment ici sur l'objectif commercial. 
19 Les séries ne sont pas l'otage d'un seul médium. Elles transmigrent désormais de la télévision à l'internet, de la $\mathrm{BD}$ au cinéma. Le commanditaire de ces différentes transmigrations sémiotiques et médiatiques est, certes, le marché. Mais le rapport que la société entretient avec les séries est-il vraiment identique à celui qu'elle entretient avec les objets de consommation? Selon Céline Masoni Lacroix et Bruno Cailler, il convient de repenser la réception des séries, en rompant avec les oppositions binaires (entre la production et la réception, entre le monde que l'on raconte et celui où l'on raconte), au profit d'une approche dialogique (au sens de Bakhtine), capable de nous faire reconnaître que certaines pratiques de réception qui semblent relever d'une phénoménologie de l'«imprégnation passive» des contenus sériels, relèvent, en réalité, d'une phénoménologie de la "transformation active » de ces mêmes contenus. Dans un environnement médiatique en pleine évolution, les publics ont, en effet, développé des compétences multiples qui redéfinissent leur rôle dans le processus d'appropriation d'un récit sériel, faisant de celui-ci le support d'une grande variété de pratiques culturelles interactives de « lecture-écriture ».

Dans l'article d'Alessandro Leiduan, la sérialité narrative est envisagée comme une notion coextensive de l'ensemble des récits - y compris de ceux qui ont vu le jour dans une époque préindustrielle. Formulée par U. Eco dans un essai de 1985, cette thèse est l'expression d'un remodelage inédit du rapport que la société entretient avec l'univers des récits - un remodelage provoqué par l'emprise que le symbolisme des objets de consommation exerce désormais sur toutes les pratiques symboliques traditionnelles. Selon l'auteur de cet article, les récits ne sont pas devenus des objets de consommation en ce sens qu'ils satisfont à certains besoins et qu'ils peuvent, de ce fait, assurer des revenus à ceux qui les produisent, les diffusent ou les mettent en vente. Les objets de consommation n'ont plus vocation à satisfaire uniquement des besoins matériels. Les significations imaginaires dont ils sont crédités par le discours publicitaire ont fait de ces objets les vecteurs d'un symbolisme tout-puissant, dont les effets se répandent bien audelà des frontières qui délimitent l'aire d'influence (hélas, de plus en plus circonscrite et asphyxiante) des pratiques symboliques traditionnelles (l'éthique, la religion, la philosophie, la politique et, bien entendu, l'esthétique). C'est en composant avec le symbolisme hégémonique des objets de consommation que les pratiques symboliques traditionnelles peuvent, dès lors, espérer prolonger leur existence dans le monde contemporain.

21 Enfin, Giuseppe Lovito étudie, dans son article, les formes de sérialité qui se fondent sur des mélanges particulièrement réussis de procédés, de topoï et de modèles narratifs et culturels conventionnels. Prenant appui sur les analyses sémiologiques d'U. Eco consacrées à la série de Superman, au roman Les Mystères de Paris de Sue et à la série de James Bond de Fleming, il montre que les auteurs de ces ouvrages se servent de ces procédés, topoï et modèles de façon sérielle et stéréotypée, dans le but d'axer leurs récits sur des héros qui, en répétant à l'identique les mêmes actions d'épisode en épisode, se laissent facilement reconnaître et apprécier par les lecteurs, qui peuvent ainsi aisément s'y identifier et être poussés à acheter la suite de leurs histoires. Toutefois, Lovito fait aussi remarquer que si, d'une part, ces stratégies permettent de cette façon de captiver et de maintenir l'intérêt des lecteurs, d'autre part, en stimulant, lors de leur lecture, des mécanismes psychologiques d'identification « consolatoire » et des processus cognitifs de décodage du "déjà connu », elles finissent de ce fait par les entretenir dans leur système 
d'idées et de valeurs consolidé, sans pour autant les amener à le mettre en quelque sorte en question.

\section{BIBLIOGRAPHY}

Benassi, S. (2000), Série et feuilletons T.V. Liège : Céfal.

Bourdieu, P. (1998), Les règles de l'art. Paris : Editions du Seuil.

Bourdieu, P. (1979), La distinction. Critique sociale du goût. Paris : Editions de minuit.

Eco, U. (1985), « L'innovazione nel seriale » dans Sugli specchi ed altri saggi. Milano : Bompiani.

Eco, U. (1983), Postille al nome della rosa. Milano : Bompiani.

Gadamer, H.-G. (1983 [1960]), Verità e metodo. Milano : Bompiani.

Genette, G. (1982), Palimpsestes. Paris : Editions du Seuil.

Kant, E. (1995 [1790]), Critique de la faculté de juger. Paris : Aubier.

Pouivet, R. (2003), L'œuvre d'art à l'âge de sa mondialisation. Bruxelles : La lettre volée.

Schaeffer, J.-M. (1999), Pourquoi la fiction? Paris : Editions du Seuil.

\section{NOTES}

1. Kant, E. (1995 [1790]), Critique de la faculté de juger. Paris : Aubier.

2. Voir, à ce sujet, chez Gadamer, la notion de «différenciation esthétique » (ästetische Unterscheidung).

3. «La révolution symbolique par laquelle les artistes s'affranchissent de la demande bourgeoise en refusant de reconnaitre aucun autre maître que leur art a pour effet de faire disparaître le marché. (...) Au moment où ils affirment, avec Flaubert, qu'“une œuvre d'art [...] est inappréciable, n'a pas de valeur commerciale, ne peut se payer", qu'elle est sans prix, c'est-à-dire étrangère à la logique ordinaire de l'économie ordinaire, on découvre qu'elle est effectivement sans valeur commerciale, qu'elle n'a pas de marché. » (Bourdieu 1998 : 139).

4. «En ce sens, la série répond au besoin infantile, mais pas nécessairement "morbide" (morboso), d'entendre encore et toujours la même histoire, d'être consolé par le "retour de l'identique » se représentant sous des formes à peine déguisées » (Eco $1985: 129$, m.t.).

5. Voir, dans ce numéro des Cahiers de narratologie, l'article de Marti.

6. Emissions de la télévision française consacrées aux faits divers criminels. 
INDEX

Chronological index: XXe siècle

Geographical index: Monde

\section{AUTHOR}

ALESSANDRO LEIDUAN

Université de Toulon, Babel, 2649 